\title{
An Asynchronous Reservation Protocol for Variable-sized Messages In Local Lightwave Networks using a Passive Star Topology
}

\author{
Jae Hwoon Lee and Chong kwan Un \\ Communications Research Laboratory \\ Department of Electrical Engineering \\ Korea Advanced Institute of Science and Technology \\ 373-1 Kusong-Dong, Yusong-Gu, Taejon 305-701, Korea \\ (TEL: +82-42-869-3415, FAX: +82-42-869-8520, E-mail: ckun@eekaist.kaist.ac.kr)
}

\begin{abstract}
This paper proposes and analyzes an asynchronous reservation protocol for a very high-speed optical LAN using a passive star topology. Each node is equipped with a single tunable transmitter and a single tunable receiver, both of which are tunable over a range of wavelengths. The protocol can efficiently support variable-sized messages, which is operated synchronously. The protocol is scalable, i.e., it can accommodate a variable number of nodes. Moreover, destination conflicts can be avoided with this protocol. Numerical results show that the asynchronous protocol achieves higher throughput than the synchronous system.
\end{abstract}

\section{INTRODUCTION}

Optical fiber is being used as a replacement of metallic cable due to the known superior characteristics for the long-haul point-to-point communication [1]. Single-mode optical fiber has a bandwidth of about $20 \mathrm{THz}$ in the low-loss region of $1.2 \mu \mathrm{m}$ to $1.6 \mu \mathrm{m}$ [2]. However, the maximum capacity of the fiber is limited by the electronic processing capability of a node of a few Gbps in a single-channel optical network. Wavelength division multiplexing (WDM) technology is a method to exploit such a huge bandwidth [3]. In WDM, the bandwidth of the fiber is divided into a set of parallel channels, each operating with different wavelength. The bandwidth of each channel is simply the transmission rate of a node. Therefore, the network using the WDM technology becomes a multichannel network. One method to employ the 
multichannel operation is that each node is equipped with a bank of transmitting lasers or filters, one for each channel. But, it is prohibitively expensive, and thus a network with a small number of nodes can only be established by using this method. Another approach is to design systems such that each node is equipped with a small number of transmitters and receivers (i.e., one or two). In this case, considering the tunability of the nodal transceivers, WDM can be classified into four systems as follows: (i) a system with fixed transmitter(s) and fixed receiver(s), (ii) a system with fixed transmitter(s) and tunable receiver(s), (iii) a system with tunable transmitter(s) and fixed receiver(s), and (iv) a system with tunable transmitter(s) and tunable receiver(s) [4]. Among these, the architecture that each node has both tunable transmitter and receiver is more favorable in accommodating a scalable node population. In this case, the transmitter of the source node and the receiver of the destination node must be tuned to the same wavelength during the message transmission time to enable the message transmission. In order for control information exchange among the nodes, a wavelength is used for the control channel and other wavelengths are used for data channels.

In [5-8], a number of random access protocols are introduced and analyzed for very highspeed optical LANs using a passive star topology. Most of the protocols are based on the transmission of fixed-sized data packets. However, when there is a need to accommodate circuit-switched traffic or traffic with long holding time (e.g., file transfers), it is necessary for a protocol to efficiently support such a variable-sized message. In [7], a buffered reservation protocol with variable message length is proposed, when the system is asynchronous. In this protocol, if a control packet is transmitted without collision, the data message is queued into the destination buffer for transmission. However, a new node cannot join the network with reinitializing the network, because of the buffered system. In [9], two reservation-ALOHA-based protocols are proposed to transmit variable-sized messages, which are operated synchronously, where all channels are slotted into cycles. These protocols can employ net nodes without network reinitialization. However, those protocols do not take destination conflicts into account. Moreover, collision at the control channel results in the waste of one cycle in a data channel, which deteriorates the performance of the network. The synchronous system also lacks the flexibility in allocating bandwidth resources.

In this paper, we propose an asynchronous reservation protocol for the transmission of variable-sized messages, where each node has only one tunable transmitter and one tunable receiver. The protocol is independent of the number of nodes and any node can determine the time of transmission of its message by itself. Also, any new node can easily join the network without network reinitialization, which acts as a node just finishing the transmission of the message. So, once a network is constructed, it is operated independently of the node insertion or deletion. Moreover, destination conflict and data channel conflict can be avoided.

The paper is organized as follows: In Section 2, we describe the details of the protocol. In Section 3, we present a performance analysis of mathematical models. Numerical results are presented in Section 4. Finally, in Section 5, we draw conclusions.

\section{ASYNCHRONOUS RESERVATION PROTOCOL}

The network architecture being considered is shown in Fig. 1. The bandwidth of the fiber is divided into $N+1 \mathrm{WDM}$ channels, each operating at a different wavelength from the set 

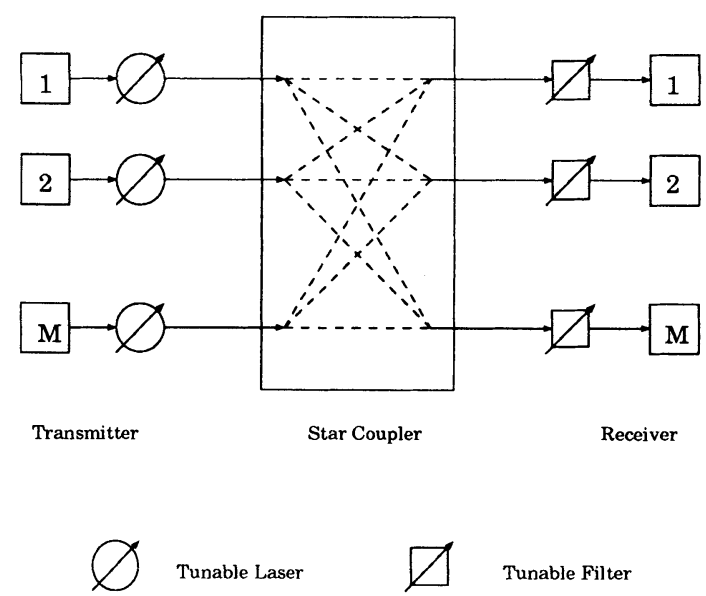

Fig. 1. Network architecture with a passive star topology.

$\left\{\lambda_{0}, \lambda_{1}, \ldots, \lambda_{N}\right\}$. There are $M(M>N)$ nodes in the network. Nodes are connected to input and output ports of a central passive star coupler, where the incident light energy from any input is equally divided among all the output ports. Thus, the star coupler acts as a broadcast medium. The star coupler is preferable because of its logarithmic splitting loss in the coupler and no tapping or insertion loss. It is assumed that each node has a tunable transmitter and a tunable receiver which can be tunable over the entire set of wavelengths utilized in the network. The tuning times of the transceiver are assumed to be zero. A receiving node needs to be informed of which channel is used in order to receive a message from a transmitting node. That is, a pretransmission coordination is required among nodes. The channel with wavelength $\lambda_{0}$ is used as a control channel for coordination of access among nodes. Those with wavelengths $\lambda_{1}, \ldots, \lambda_{N}$ are used as data channels for actual message. Control packets are transmitted through the control channel which are used for signaling between nodes and for reserving data channels during the message transmission time. A control packet contains the addresses of source and destination nodes and the wavelength to be used for the transmission of the actual message. The wavelength is randomly chosen by a source node before the node monitors the control channel, which is called the random selection scheme.

The proposed protocol is operated asynchronously. That is, data channels are not slotted and the control channel is slotted with the duration of transmission time of the control packet. The control channel is shared by all nodes on a contention basis using the slotted ALOHA protocol. The data packet has the same length as the control packet, and is grouped into messages of random size. All nodes are assumed to be $D$ slots apart. Therefore, a traffic transmitted by a node is received by all nodes which monitor the control channel by way of the star coupler, $D$ slots later. When a control packet arrives at the destination node whose receiver is not tuned to the control channel or when there may be more than one such message arriving within the message transmission time, destination conflicts may happen [10]. Moreover, data channel conflicts may occur when more than one source node try to use the same data channel at the 
same time. Thus, any source node should employ the following message transmission procedure in order to transmit a message without data channel and destination conflicts.

\section{Message Transmission Procedure}

\section{(1) Local Control Channel Monitoring(LCCM)}

Assume that a message (new or retransmitted) is generated at node $i$ to be destined to node $j$, at slot $t$. Node $i$ chooses one of the data channels randomly, say $n$. The node monitors the control channel during $K$ consecutive control slots following the message generation time. $K$ is called the local monitoring period which is an integer value that is not less than $\max (N, D+1)$. Node $i$ then checks the following conditions:

(a) No control packet is observed at slot $(t+D+1)$.

(b) No control packet to $j$ is observed during $K$ slots.

(c) No control packet to $i$ is observed during $K$ slots.

(d) No control packet with the $n$th data channel number is observed during $K$ slots.

If any of the above conditions are not satisfied, the transmission procedure at node $i$ will be restarted after a random back-off period. Otherwise, the node goes to the next state.

(2) Control Packet Transmission(CPT)

Node $i$ transmits its control packet over the control channel in slot $(t+K+1)$, and continues to the next state. In this case, the control packet is called the control packet for signaling (S_CP).

(3) Data channel and Destination Conflicts Detection(DCDCD)

There is a $D$ slot period between the transmission of a control packet by a node and prior to its return. Node $i$ monitors the control channel during slot $(t+K+1)$ through slot $(t+K+D)$, to check whether a control packet with $n$th data channel or a successful control packet to node $j$ or $i$ exists during the propagation delay. If such a control packet is received, node $i$ restarts from the LCCM after the random back-off period. Otherwise, the node continues to the next state.

(4) Control Packet Collision Detection(CPCD)

The control packet transmitted in slot $(t+K+1)$ is detected by all nodes that monitor the control channel in slot $(t+K+D+1)$. If the control packet is returned to node $i$ without collision, the node considers to succeed in reserving $n$th data channel, tunes its transmitter to the selected data channel, and goes on the next state. The destination node will also tune its receiver to the intended wavelength. Otherwise, after a random back-off time, the transmission procedure restarts from LCCM.

(5) Data Message Transmission(DMT)

If the length of a message is not greater than $K$, node $i$ transmits its message through the reserved data channel. Moreover, the node returns to LCCM and waits for another message. Otherwise, the node transmits $(K-1-D)$ data packets among the message. And then, the node tunes its transmitter to the control channel immediately, and sends the control packet. In this case, the control packet is called the control packet for reserving (R_CP). The node repeats the transmission of $(K-1)$ data packets among the remaining message through the reserved data channel and the transmission of the control packet 


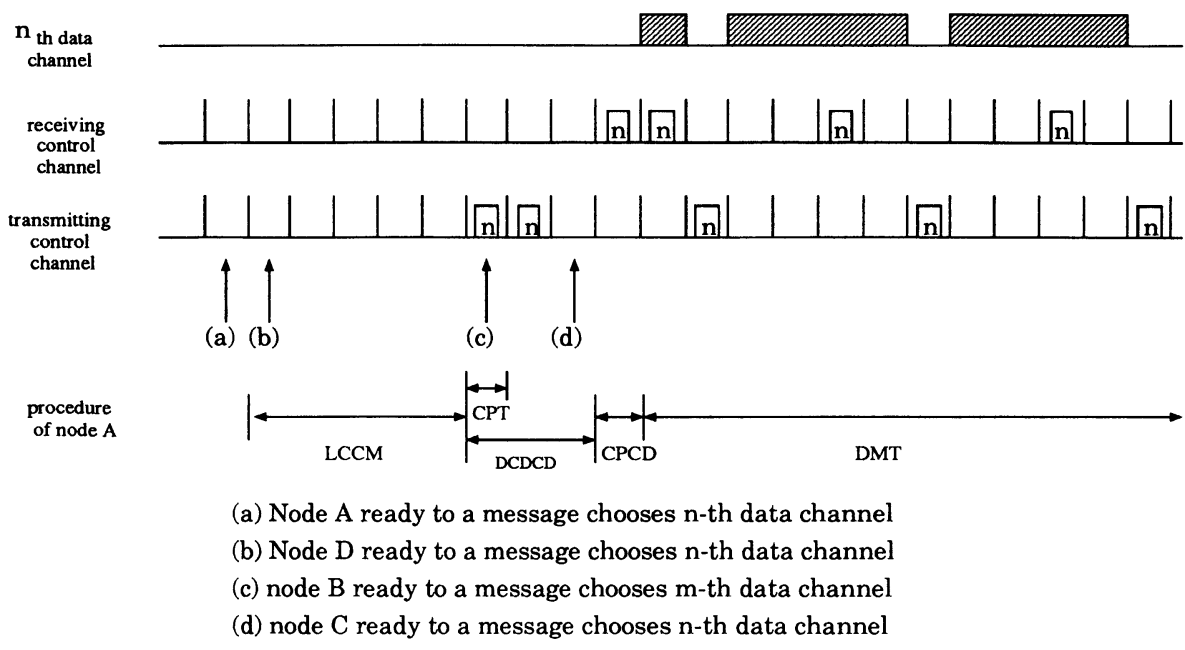

Fig. 2. Message transmission procedure $\mathrm{K}=5$ and $\mathrm{D}=3$.

through the control channel until the length of the remaining message is not greater than $(K+D)$. If the length of the remaining message is not greater than $(K+D)$, the node sends all data packets of the remaining message, and goes to LCCM.

Fig. 2 shows an example of the message transmission procedure of the asynchronous reservation protocol. When a message is generated at node $A$ in slot $t$, the node acquires $n$th data channel successfully, according to the above procedure. When observing the control channel, control packets transmitted by node $A$ are presented in every $K$ slots. Such a control packet is preserved by a node which tries to reserve a data channel by condition (a) in LCCM. That is, node $B$ transmits its S_CP, then the S_CP would be collided with R_CP of node $A$. Node $C$ defers the transmission of its S_CP because of selecting the same data channel that node $A$ uses. So, the node which has successfully acquired a data channel exclusively uses the channel until the transmission of its message is finished. Node $D$ considers its S_CP as failure because the node chooses the same data channel of node $A$ 's, although the S_CP is successfully transmitted through the control packet.

\section{PERFORMANCE ANALYSIS}

Now, we analyze the performance of the asynchronous reservation protocol. As described before, destination conflicts may occur when more than one source nodes try to communicate with a destination node at the same time. However, the performance deterioration due to destination conflicts is small when the number of nodes is much greater than that of wavelengths, because the probability that more than one source nodes choose the same destination node is negligible [5]. Therefore, we assume an infinite number of nodes which collectively form an independent 


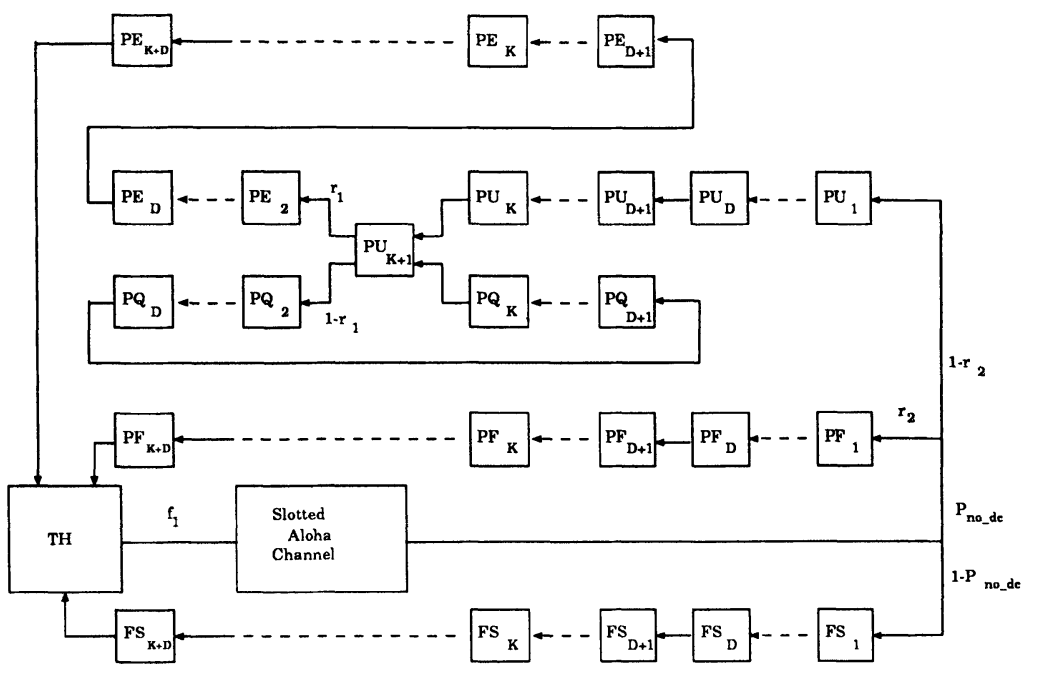

Fig. 3. Analytic model for the asynchronous reservation protocol.

Poisson source with an aggregate mean packet traffic rate of $g$ (messages/slot). When observing the control channel, control packets for signaling messages (new and retransmitted) are generated according to Poisson process with parameter $G$ (messages/slot). Each message arrived at a node is assumed to be geometrically distributed with mean $1 / p$ (slots). An analytic model for the asynchronous reservation protocol is shown in Fig. 3. We assume that the transitions of nodes from one mode to another occur at the end of each slot and that the model is formulated by observing the system state only at the beginning of each slot. Each node can be in one of the following $(5 K+3 D-1)$ modes: $T H, P F_{i}(1 \leq i \leq K+D), F S_{j}(1 \leq j \leq K+D)$, $P U_{k}(1 \leq k \leq K+1), P Q_{l}(1 \leq l \leq K-1)$, and $P E_{m}(2 \leq m \leq K+D)$. All modes except for the $T H$ (thinking) mode are unit delay elements, and represent the influence of channel propagation delay and/or local monitoring period. A node in $P F_{i-1}$ will move into $P F_{i}$ in the next slot with probability 1 . The same also applies to $F S_{j}, P U_{k}, P Q_{l}$ and $P E_{m}$ modes. There are an infinite number of nodes in the $T H$ mode. When a node with a message does not satisfy the conditions in the LCCM state, the node defers the transmission of its S_CP and enters the $T H$ mode again. Moreover, when more than one S_CP are transmitted in a slot, collision of control packets occurs. Any node having control packet collision also enters the $T H$ mode. Let $f_{1}$ be the probability that a single S_CP $_{-}$is generated and transmitted according to the CPT state and it does not incur control packet collision. In the following, S_CP means the control packet generated according to the probability $f_{1}$, which does not collided with others. There is only one node in all modes except for the $T H$ mode, because only one control packet can be generated according to $f_{1}$. Due to the propagation delay, there may be many uncollided S_CPs with the same data channel number within a $D$-slot period. In this case, the other control packets except for the first one are considered to be failed in reserving a data channel, in order to avoid data channel conflict. Let $P_{n \dot{u}_{-} d c}$ be the probability that an S_CP does not incur the data channel 
conflict. Then, with the probability $\left(1-P_{n o_{-} d c}\right)$, the node having transmitted its S_CP enters $F S_{1}$ mode. The node having transmitted its $\mathrm{S}_{-} \mathrm{CP}$ in $F S_{1}$ finds the failure of its control packet in $F S_{D+1}$ mode. A node with a message may not transmit its S_CP because the node finds the control packet in $F S_{D+1}$ mode due to the condition (a) in the LCCM state, even though the control packet in $F S_{D+1}$ fails in reserving a data channel.

Let $r_{2}$ be the probability that the length of the message is not greater than $K$. It is given by the following:

$$
r_{2}=\sum_{k=1}^{K}(1-p)^{k-1} p=1-(1-p)^{K} .
$$

Then, the node succeeded in reserving a data channel enters either $P F_{1}$ mode with probability $r_{2}$, or $P U_{1}$ mode with $1-r_{2}$. The node having transmitted its S_CP in $P F_{1}\left(P U_{1}\right)$ finds the success of its control packet in $P F_{D+1}\left(P U_{D+1}\right)$ mode, and the one at $P F_{D+2}$ mode starts to transmit one data packet per one slot till its message is completely transmitted, and then it enters the $T H$ mode. Let $S_{2}$ be the average length of one message that a node in $P F_{D+2}$ transmits. Then $S_{2}$ can be calculated as follows.

$$
S_{2}=\frac{1-(K+1)(1-p)^{K}+K^{r}(1-p)^{K+1}}{p r_{2}} .
$$

The node in $P U_{j}(D+2 \leq j \leq K)$ transmits one data packet among its message, and the one in $P U_{K+1}$ mode transmits its R_CP. The length of the remaining message in the node just after having the transmission of R_CP is always greater than $(D+1)$. Let $r_{1}$ be the probability that the remaining message is not greater than $(D+1+K-1)$. It is given as follows:

$$
r_{1}=\sum_{k=1}^{K-1}(1-p)^{k-1} p=1-(1-p)^{K-1} .
$$

Then, the node in $P U_{K+1}$ mode moves into either $P E_{2}$ mode with probability $r_{1}$ or $P Q_{2}$ mode with $\left(1-r_{1}\right)$. The node in $P Q_{l}(2 \leq l \leq K)$ mode transmits one data packet among its message. A node in $P E_{2}$ transmits all of its remaining message and goes to the $T H$ mode. Let $S_{1}$ be the average length of the remaining message that a node in $P E_{2}$ transmits. Then $S_{1}$ can be calculated as the following:

$$
S_{1}=D+1+\frac{1-K(1-p)^{K-1}+(K-1)(1-p)^{K}}{p r_{1}} .
$$

There is only one node in either $P F_{i}, F S_{j}, P U_{k}, P Q_{l}$ or $P E_{m}$ mode, as explained earlier. Moreover, nodes in $P F_{i}, P U_{k}, P Q_{l}$ and $P E_{m}$ modes use different data channels. Nodes in $F S_{j}$ have the same data channels which are used by nodes in $P F_{i}, P U_{k}, P Q_{l}$ and $P E_{m}$.

We now define a state vector of the system. The system state in a slot $t$ can be described by the following vector:

$$
\pi(t)=\left(N_{P F_{i}}(t), N_{F S_{j}}(t), N_{P U_{k}}(t), N_{P Q_{l}}(t), N_{P E_{m}}(t)\right)
$$

where $N_{X}(t)$ is the number of nodes in mode $X$ at time $t$ with $X$ having one of the $(5 K+3 D-1)$ modes described above. Then the vector $\pi(t)$ forms a discrete-time Markov chain. But, its state space is large, and thus is very complex to solve. Therefore, we utilize the equilibrium point 
analysis (EPA) method [11] to analyze this chain. An equilibrium point is defined as a point at which the expected increase in the number of nodes in each mode in a unit time is zero. In this method, we assume that the system is always at an equilibrium point. Applying the condition to all the modes. we get a set of equations called the equilibrium point equations, the solution of which yields one or more equilibrium points. The system is said to be stable if the number of solutions is one; otherwise, the system is said to be unstable.

Let $\bar{\pi}=\left(\bar{N}_{F S_{i}}, \bar{N}_{P F_{i}}, \bar{N}_{P U_{i}}, \bar{N}_{P Q_{i}}, \bar{N}_{P E_{i}}\right)$ be an equilibrium point, where $\overline{N_{X}}$ is the number of nodes in mode $X$ in the steady state. Let $\delta_{X}(\bar{\pi})$ be the expected increase in the number of nodes in mode $X$ in a single transition, given that the system is at $\bar{\pi}$. Since $\delta_{F S_{i}}(\bar{\pi})=\bar{N}_{F S_{i-1}}-\bar{N}_{F S_{i}}=0(i=2, \ldots, K+D)$ under the equilibrium point $\bar{\pi}$, we have

$$
\bar{N}_{F S_{i}}=f_{1}\left(1-P_{n o_{-} d c}\right), 1 \leq i \leq K+D \text {. }
$$

Similarly,

$$
\begin{aligned}
\bar{N}_{P F_{i}} & =r_{2} f_{1} P_{n o_{-} d c}, 1 \leq i \leq K+D \\
\bar{N}_{P U_{i}} & =\left(1-r_{2}\right) f_{1} P_{n o_{-} d c}, 1 \leq i \leq K \\
\bar{N}_{P U_{K+1}} & =\frac{1-r_{2}}{r_{1}} f_{1} P_{n u_{-} d c} \\
\bar{N}_{P Q_{i}} & =\frac{\left(1-r_{1}\right)\left(1-r_{2}\right)}{r_{1}} f_{1} P_{n o_{-} d c}, \quad 2 \leq i \leq K \\
\bar{N}_{P E_{i}} & =\left(1-r_{2}\right) f_{1} P_{n o_{-} d c}, \quad 2 \leq i \leq K+D .
\end{aligned}
$$

Let $T$ be the number of different data channels that a node can know from the control packets by observing the control channel during a $K$-slot period, when the node is in the LCCM state. Then, $T$ can be calculated as follows.

$$
\begin{aligned}
T & =\sum_{k=2}^{K+D} \bar{N}_{P E_{k}}+\sum_{k=2}^{K} \bar{N}_{P Q_{k}}+\sum_{k=D+1}^{K+1} \bar{N}_{P l_{k}}+\sum_{k=D+1}^{K+D} \bar{N}_{P F_{k}} \\
& =K\left\{2-r_{2}+\frac{\left(1-r_{1}\right)\left(1-r_{2}\right)}{r_{1}}\right\} f_{1} P_{n u_{-} d c} .
\end{aligned}
$$

The node considers that $T$ among $N$ data channels are used by nodes in the DMT state. If the node chooses one of $(N-T)$ data channels, the node transmits its S_CP. And the node monitors the control channel during $D$ slots (DCDCD state). Let $W$ be the number of different data channels that a node can know from the control packets received by observing the control channel during a $D$-slot period, when the node is in the DCDCD state. Then, $W$ can be obtained as follows.

$$
W=\sum_{k=1}^{D}\left(\bar{N}_{P U_{k}}+\bar{N}_{P F_{k}}\right)=D f_{1} P_{n c_{-} d c} .
$$

The node also considers that $W$ among $N-T$ data channels are newly reserved. If the data channel selected by the node is one of the $W$ data channels, the node considers that its S_CP has failed in reserving the data channel. Otherwise, the node considers that it has reserved the data channel successfully. Therefore, $P_{n o_{-} d c}$ is calculated as

$$
P_{\text {noddc }}=1-\frac{W}{N-T} .
$$


Therefore, if $f_{1}$ can be obtained, we can calculate $P_{n o_{-} d c}$ and also obtain the state of all modes. Let $U$ be the probability that there exists a node in either $F S_{K}, P F_{K}, P Q_{K}, P U_{K}$ or $P E_{K} . U$ is equal to the probability that a node ready for a message in slot $t$ finds a control packet in slot $(t+D+1)$, which can be obtained as

$$
U=\bar{N}_{F S_{K^{*}}}+\bar{N}_{P F_{K^{*}}}+\bar{N}_{P Q_{K}}+\bar{N}_{P U_{K^{*}}}+\bar{N}_{P E_{K^{*}}} .
$$

Then, $f_{1}$ can be obtained as

$$
f_{1}=(1-U) \frac{N-T}{N} G e^{-\frac{N-T}{N} G} .
$$

From (10) and (12), we can obtain the equilibrium point $\bar{\pi}$ by solving the nonlinear equation about $f_{1}$.

Network throughput, $S_{N T H}$, is defined as the conditional expectation of the number of successfully transmitted data packets in a slot, given that the system is in state $\bar{\pi}$. Then, it is clear that the throughput at an equilibrium point is expressed as

$$
\begin{aligned}
S_{N T H} & =S_{2} \bar{N}_{P F_{K+D}}+(K-D-1) \bar{N}_{P U_{K}}+(K-1) \bar{N}_{P Q_{2}}+S_{1} \bar{N}_{P E_{K^{\prime}+D}} \\
& =\left\{S_{2} r_{2}+\left(1-r_{2}\right)\left(S_{1}-D+\frac{K-1}{r_{1}}\right)\right\} f_{1} P_{n u_{-} d c} .
\end{aligned}
$$

Normalized per channel throughput, $S_{d}$, can be obtained as

$$
S_{d}=\frac{1}{N+1} S_{N T H}
$$

\section{NUMERICAL RESULTS}

In this section, we investigate the performance of the proposed protocol with various system parameters. Simulation was done to test the accuracy of the analytic results. As will be shown, in general the analytic results obtained by the EPA method agree closely with simulation results. But these results become deviated and the throughput usually takes the maximum value near the point where the network becomes unstable [11]. Moreover, the results become generally inaccurate when the network is unstable. When the network is unstable (bistable), the analytic results corresponding to two locally stable equilibrium points are depicted.

Fig. 4 shows the network throughput versus offered load for various numbers of data channels, and Fig. 5 shows the normalized per channel throughput under the same condition as in Fig. 4. With a low load, any node which tries to transmit its message transmits its S_CP according to the message transmission procedure, and the control packet may be sent without collision. Meanwhile, nodes having reserved data channels send their own R_CPs every $K$ slots. When observing the control channel, the number of control packets, which may appear during $K$ consecutive slots, increases, as $N$ increases. In other words, when a message arrives at a node in slot $t$, the probability that the node encounters the control packet in slot $(t+D+1)$ is greater at higher $N$. Therefore, as $N$ increases, $S$ also increases, but $S_{d}$ decreases. As the load 


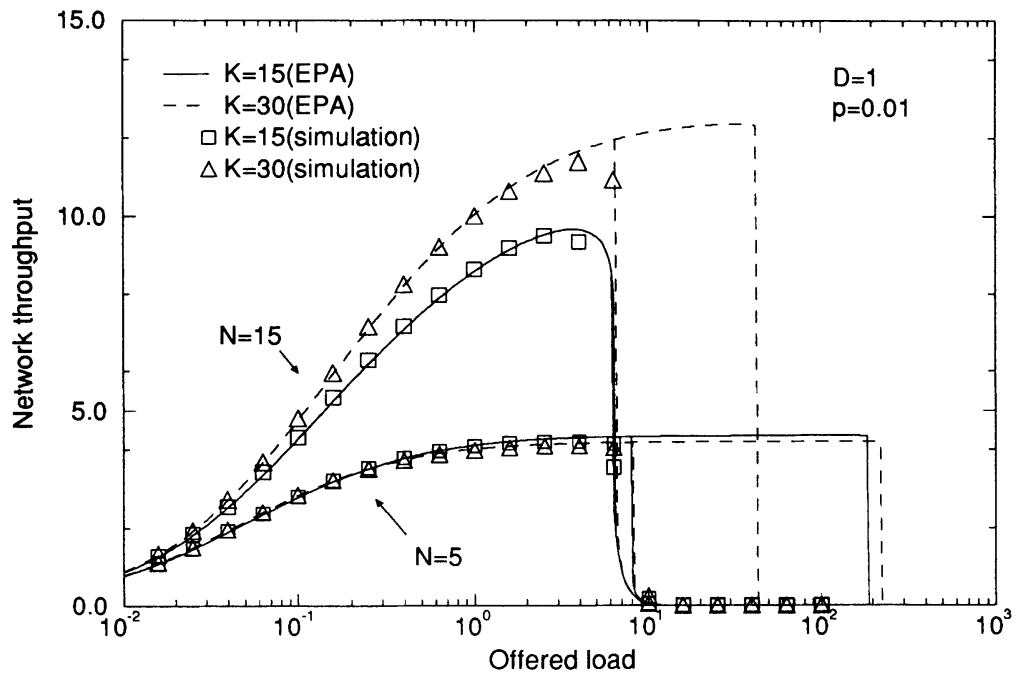

Fig. 4. Network throughput versus offered load.

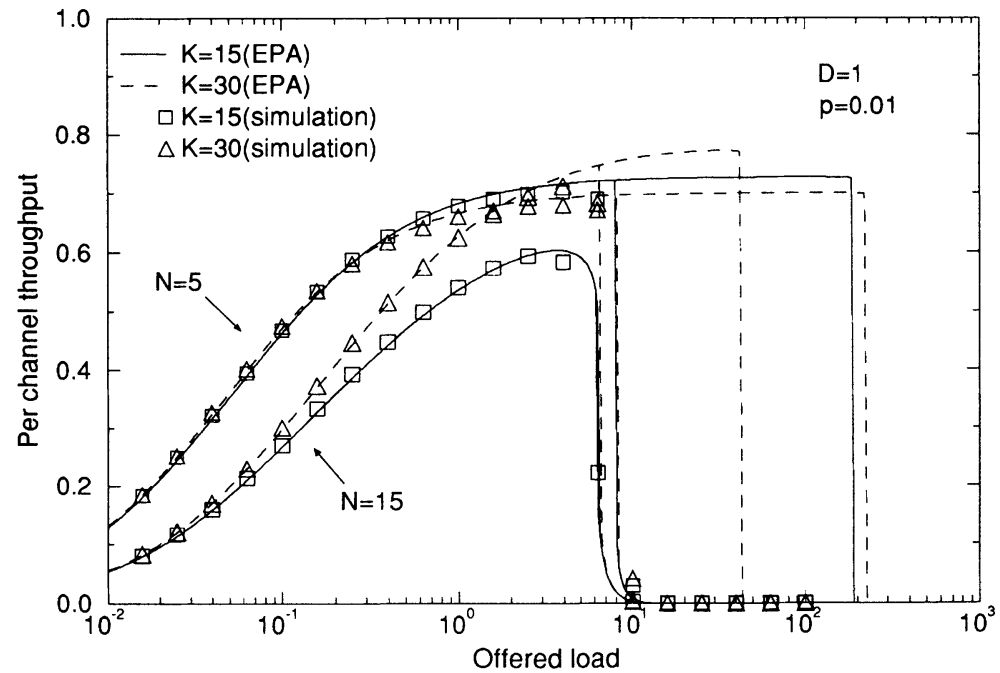

Fig. 5. Normalized per channel throughput versus offered load.

increases, the number of nodes which are ready to transmit their messages also increases. Hence the probability of control packet collision may increase in the case of lower $N$. It is because the number of nodes choosing the same data channel increases, as $N$ decreases. 


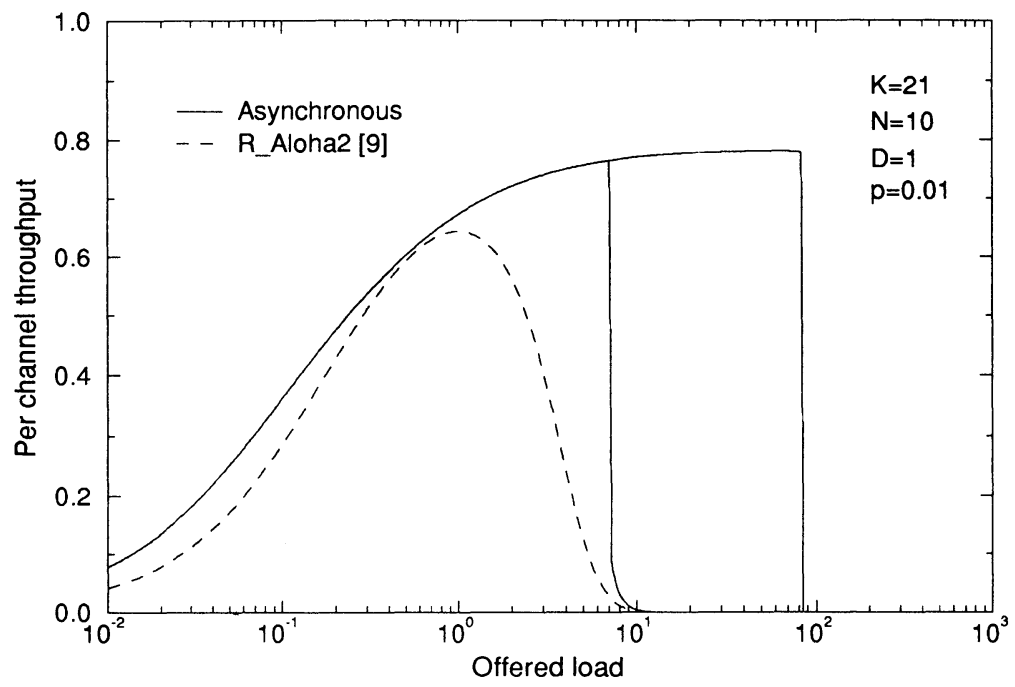

Fig. 6. Normalized per channel throughput versus offered load.

In Fig. 6, we plot the normalized per channel throughput versus offered load and R_Aloha case 2 scheme of [9] for comparison. The normalized per channel throughput equation for the R_Aloha scheme is modified with the notation used in this paper, and is given as

$$
S_{R_{-} \text {Aloha }}=\frac{N}{N+1} \frac{K-1}{K} \frac{G e^{-G} \frac{1 / p}{K-1}}{1+G \epsilon^{-G}\left(\frac{1 / p}{K-1}-1\right)} .
$$

The R_Aloha protocol is a synchronous system in which all channels are divided into 'cycle'. In this protocol control slots are preassigned to data channels. When $G$ is greater than 1.0 , more than one control packets are likely to be generated in one control slot, thus resulting in control packet collision when the corresponding data channel is idle. The control packet collision results in the waste of one cycle in a data channel, where the length of one cycle is much greater than the length of the control packet. When $G$ is less than 1.0, no control packet is likely to be generated in one control slot, which also results in waste of one cycle. On the other hand, in the case of the asynchronous reservation protocol, control packet collision or no control packet generation only wastes just as much as the length of one control packet. Therefore, the asynchronous scheme yields higher throughput than the R_Aloha scheme.

Fig. 7 presents the throughput versus local monitoring period for various numbers of data channels. As explained earlier, any node which tries to its S_CP may frequently encounter a control packet for a small $K$, which lowers the throughput. On the other hand, for a large $K$, as $K$ increases, the throughput also decreases. The reason is as follows. Let $\mathrm{rm}$ be the length of the remaining message after having transmitted the control packet. If $\mathrm{rm}$ is not greater than $(K+D)$, then $(K+D-r m)$ slots may be wasted in a data channel after finishing the transmission of the message. Therefore, the number of wasted slots increases, as $K$ increases. Thus, it is necessary to determine the value of $K$ in order to obtain the maximum throughput. 


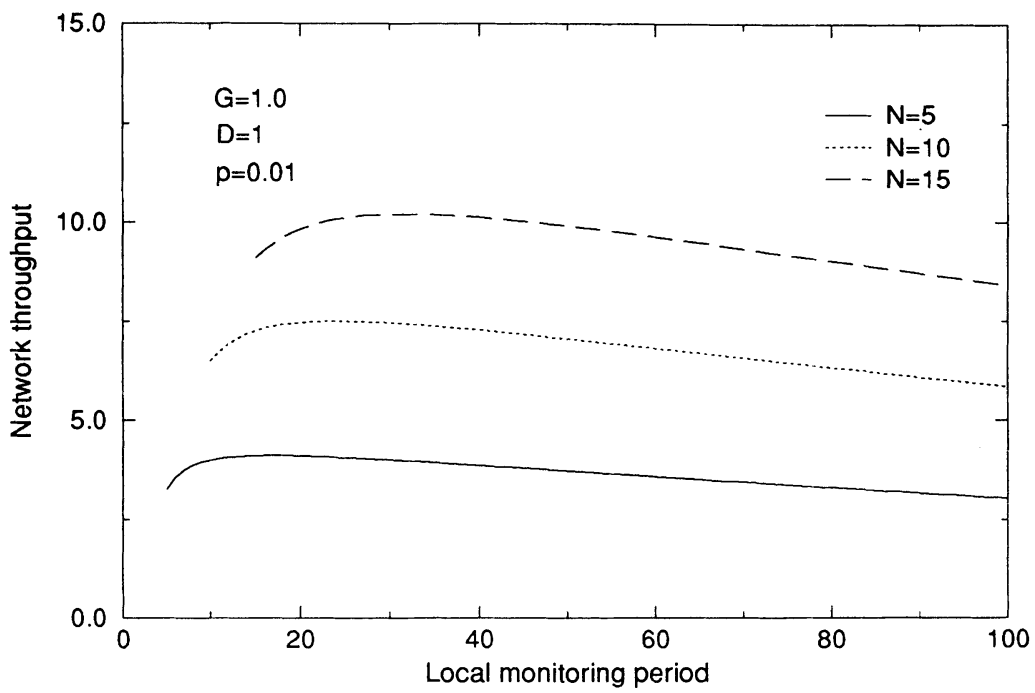

Fig. 7. Network throughput versus local monitoring period.

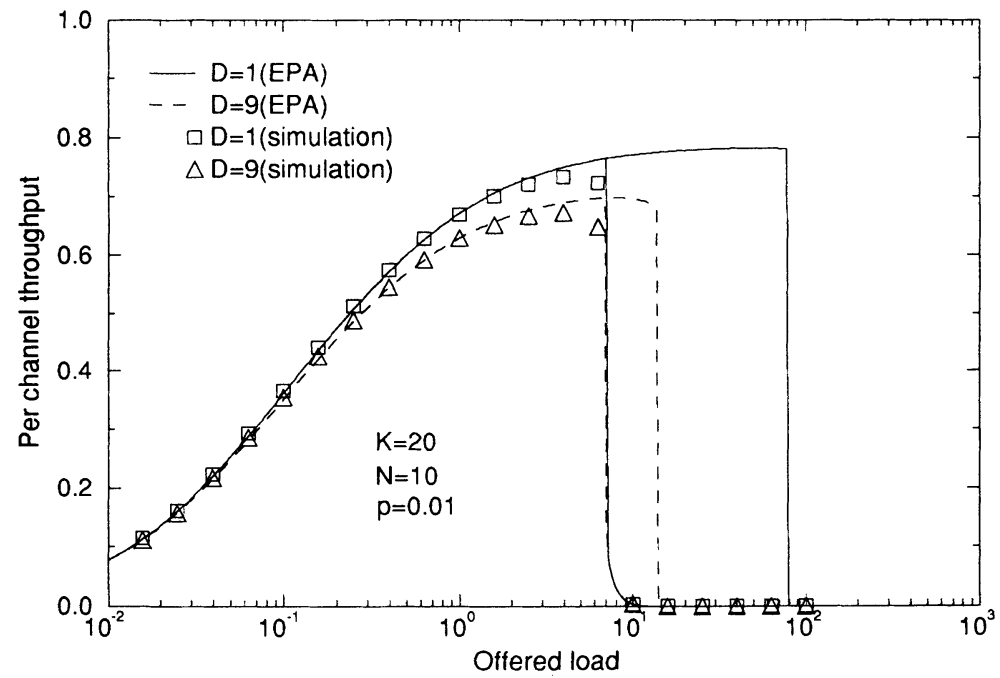

Fig. 8. Normalized per channel throughput versus offered load.

Fig. 8 shows throughput versus offered load for various end-to-end propagation delays. When a node sends its control packet in slot $t$, the node receives the control packet in slot $(t+D+1)$. Another node may transmit its control packet in slot $t^{\prime}\left(t<t^{\prime}<t+D\right)$, which 


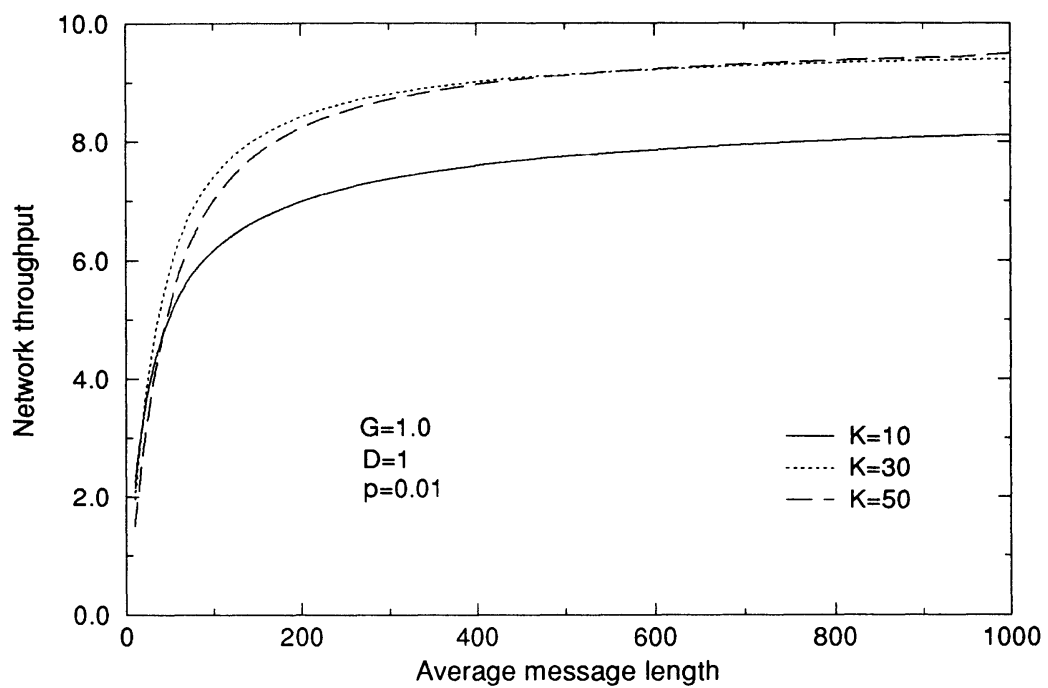

Fig. 9. Netwotk throughput versus average message length.

has the same data channel number as that of the control packet transmitted in slot $t$. Then, the control packet transmitted in slot $t^{\prime}$ is considered as a failure because of the data channel conflict. However, it may collide with another control packet which may otherwise become a "successful" control packet. Such a phenomenon may occur frequently as the propagation delay increases, which deteriorates the throughput.

Fig. 9 shows the throughput versus average message length for various values of $K$. If the message is relatively short, the number of wasted slots is increased for large $K$. Once a node successfully reserves a data channel, the message from the node is transmitted without data channel conflicts. Therefore, as the length of the message increases, the throughput also increases.

\section{CONCLUSION}

In this paper, we have presented and analyzed an asynchronous reservation protocol for highspeed local area networks using a passive star topology. Each node is only equipped with a single tunable transmitter and a single tunable receiver, both of which are tunable over the entire set of wavelengths. A control channel is used for the exchange of the control traffic. A random scheme has been considered as the data channel selection strategy. The performance of the protocol has been analyzed for the infinite population case by using the EPA method. The analytic results have been verified by comparing with simulations. Also, the effects of various system parameters have been investigated. The results shows that the protocol can yield high system throughput. This protocol is suitable for the network that requires a need to accommodate variable-sized messages such as circuit-switched traffic or traffic with long holding times (e.g., 
file transfers). The protocol is operated asynchronously, and enables any new node to join the network without network reinitialization.

\section{REFERENCES}

[1] G R Hill, "Wavelength Domain Optical Network Techniques", IEEE Proc., vol. 77, No. 1, pp. 121-132, Jan. 1990.

[2] L. G. Razowsky, C. Barry, M. Hichey, C. A. Noronha Jr. and P. Poggiolini, "WDM Local Area Networks", IEEE LTS., pp. 8-15, May 1992.

[3] S. S. Wagner and H. Kobrinski, "WDM Application in Broadband Telecommunication Networks", IEEE Commun. Mag., vol. 17, pp. 22-30, Mar. 1989.

[4] B. Mukherjee, "WDM-Based Local Lightwave Networks Part I: Single-Hop Systems", IEEE Network Mag., pp. 12-27, May 1992.

[5] I. M. I. Habbab, M. Kavehrad and E. W. Sundberg, "Protocols for Very High-Speed Optical Fiber Local Area Networks Using a Passive Star Topology", IEEE J. Lightwave Technol., vol. LT-S, pp. 1782-1794, Dec. 1987.

[6] N. Mehravari, "Performance and Protocol Improvements for Very High Speed Optical Fiber Local Area Networks Using a Passive Star Topology", IEEE J. Lightwave Technol., vol. 8, pp. 520-530, Apr. 1990.

[7] H. B. Jeon and C. K. Un, "Contention-Based Reservation Protocols in Multiwavelength Optical Networks with a Passive Star Topology", IEEE Trans. on Commun. (to appear).

[8] M. S. Chen, N. R. Dono and R. Ramaswami, "A Media-Access Protocol fpr PacketSwitched Wavelength Division Multi-Access Metropolitan Area Networks", IEEE J. Select. Areas Commun., vol. 8, pp/ 1048-1057, Aus. 1990.

[9] G. B. M. Sudhakar, N. Georganas and M. Kavehrad, "Slotted Aloha and Reservation Aloha Protocols for Very High-Speed Optical Fiber Local Area Networks Using Passive Star Topology", IEEE J. Lightwave Technol., vol. 9, no. 10, pp. 1411-1422, Oct. 1991.

[10] F. Jia and B. Mukherhee, "The Receiver Collision Avoidance (RCA) Protocols for a Single-Hop WDM Lightwave Networks", Proc. ICC , pp. 6-10, 1992.

[11] S. Tasaka, Performance Analysis of Multiple Access Protocols, MIT Press, 1986. 


\section{BIOGRAPHY}

Jae hwoon Lee was born in Seoul Korea, on 11 October 1962. He received the B.S. degree in electronic engineering from Hanyang University, Seoul, Korea, in 1985, and the M.S. degree in electrical engineering from Korea Advanced Institute of Science and Technology (KAIST), in 1987. He is currently working on the Ph.D. degree in electrical engineering at KAIST.

During 1987-1990, he served as a Researcher in the DACOM. At DACOM, his research area was line of packet switching network protocols. His research interests include performance analysis, wavelength division multiple access networks and high-speed networks.

Chong Kwan Un was born in Seoul, Korea. He received the B.S., M.S., and Ph.D. degrees in electrical engineering from the University of Delaware, Newark, in 1964, 1966, and 1969, respectively.

From 1969 to 1973 he was an Assistant Professor of Electrical Engineering at the University of Maine, Portland, where he taught communications and did research on synchronization problems. In May 1973 he joined the staff of the Telecommunication Sciences Center, SRI International, Menlo Park, CA, where he did research on voice digitization and bandwidth compression systems. Since June 1977 he has been with Korea Advanced Institute of Science and Technology (KAIST), where he is a Professor of Electrical Engineering, teaching and doing research in the areas of digital communications and signal processing. So far, he has supervised $51 \mathrm{Ph} . \mathrm{D}$. and more than $100 \mathrm{M}$.S. graduates. He has authored or coauthored over 300 papers on speech coding and processing, adaptive signal processing, data communications, B-ISDN, protocol design and analysis, and very high-speed packet communication systems. Also, he holds seven patents granted. From February 1982 to June 1983 he served as Dean of Engineering at KAIST.

Dr. Un is a Fellow of IEEE. He received a number of awards, including the 1976 Leonard G. Abraham Prize Paper Award from the IEEE Communications Society, the National Order of Merits from the Government of Korea, and Achievement Awards from the Korea Institute of Telematics and Electronics, the Korea Institute of Communication Sciences, and the Acoustical Society of Korea (ASK). He was President of the ASK from 1988 to 1989. He is a member of Tau Betaa Pi and Eta Kappa Nu. 